\title{
Size of the Observable Universe
}

\author{
Paul Halpern and Nick Tomasello \\ Dept. of Math, Physics, and Statistics, University of the Sciences in Philadelphia, 600 S. 43rd St., Philadelphia, \\ PA, United States \\ Email: p.halper@usciences.edu
}

\begin{abstract}
Because of the expansion of space and the finite age of the cosmos, there exists a horizon beyond which the light emitted by objects will never be able to reach us, marking the bounds of the observable universe. One can calculate the current distance to the horizon by tracing the amount of time it would have taken a photon starting from an object currently there to have reached us. In a 2005 paper, Gott et al. derived this distance using first year CMB data from the WMAP survey. However, more recent CMB data collected by the Planck satellite and published in 2013 have since yielded different values of cosmological parameters. In this paper, we have applied these updated parameters to refine the distance to the edge of the observable universe. We have determined a revised value for the radius of the observable universe that is $0.7 \%$ smaller than the previous estimate.
\end{abstract}

Keywords: Hubble radius; particle horizon; conformal time

\section{Introduction}

For as long as humans have been gazing at the night sky, the question of how far we can see into it has fascinated astronomers. In the nineteenth century, Heinrich Olbers called attention to the issue in his famous paradox: assuming that the universe is both static and infinitely old, and that light is our most effective vehicle of observation, how is it that the night sky takes the form of a dark canvas spotted with stars? According to Olbers, if these assumptions were true, then the sky should perpetually be filled with bright light, as there would have been enough time in the history of the universe for photons from every star in existence to have reached us.

Thankfully, astronomical revelations of the past century have unraveled Olbers' Paradox. The conundrum was resolved with the discoveries that the universe is not infinitely old but instead has a measurable age and that the cosmic fabric is not static. Because the universe is expanding the sphere of what we can see is not defined simply by the age of the universe multiplied by the speed of light. That is because the universe is expanding: the distance between every point of the fabric of spacetime is forever increasing, a growth that affects the paths of photons.

Furthermore, as two teams headed by Riess et al. [1] and Perlmutter et al. [2] have shown, using type Ia supernovae as standard candles, space is not just expanding but accelerating in its expansion. Thus, although the longest amount of time that photons can have traveled through spacetime is 13.8 billion years (the estimated age of the universe), the objects that emitted them must be farther than 13.8 billion lightyears away, since they would maintain an overall outward radial motion due to space's expansion.

An important implication of this conclusion is that there are bounds to what we can observe. However, common misconceptions persist about the extent of these bounds, several of which were addressed by Davis and Lineweaver [3]. Included in their discussion is a dispelling of the idea that the farthest we can see is defined by the Hubble radius, a value close to 13.8 billion light-years. As Davis and Lineweaver point out, the boundary of the observable universe is actually the particle horizon, the distance to which is given by the conformal time multiplied by the speed of light.

An estimate of the distance to this particle horizon, the current edge of the observable universe, was calculated by Gott et al. [4] and published in the seminal paper "A Map of the Universe." Using then-current first year data from the surveys of the WMAP satellite [5], and assuming a flat $(k=0)$ universe, Gott's team integrated the first Friedman equation to find a general expression for the conformal time at a given scale factor $a$. Taking into account the universe's current scale factor, this yielded a value 
of about $14,300 \mathrm{Mpc}$ for the distance to the particle horizon assuming that expansion began at the instant of the Big Bang. Current telescopes collect photons, however, which would not have been able to travel freely until after the recombination era. Calculating from the end of recombination, Gott's team attained the more realistic value of $14,000 \mathrm{Mpc}$.

While the value of 14,000 Mpc remains a solid estimate today, new cosmological data provided by the Planck satellite in 2013 [6] suggests the need for an updated calculation. Using this 2013 data, which includes updated values for the current scale factor $a_{0}$, the Hubble radius $R_{H 0}$, and the various density parameters connected with matter, radiation, and dark energy, an accurate distance to the edge of the observable universe may be arrived at by finding the current value of the conformal time. This distance can then be even further refined by recalculating the conformal time using the value provided by Planck for the redshift at the moment of recombination, $z_{\text {rec }}$.

\section{Calculating the Co-moving Radius}

The current radius of the observable universe can be calculated by following the procedure of Gott et. al. Choosing units in which $G=c=1$, the first Friedman equation becomes:

$$
\left(\frac{\dot{a}}{a}\right)^{2}=\frac{-k}{a^{2}}+\frac{\Lambda}{3}+\frac{8 \pi \rho_{m}}{3}+\frac{8 \pi \rho_{r}}{3}
$$

where $\rho_{m}$ and $\rho_{r}$ are the densities of matter and radiation respectively.

The conformal time $\eta(t)$ is related to the cosmological time $t$ by the expression:

$$
\eta(t)=\int_{0}^{t} \frac{\mathrm{d} t}{a}
$$

Rearranging expression [1] in terms of $\mathrm{d} t$ and inserting into the integral [2] we obtain:

$$
\eta(t)=\int_{0}^{t}\left[-k a^{2}+\frac{a^{4} \Lambda}{3}+\frac{a^{4}\left(\rho_{m}+\rho_{r}\right)}{3}\right]^{-1 / 2} \mathrm{~d} a
$$

From the 2013, we insert the various cosmological parameters:

$H_{0}=67.3 \mathrm{~km} \mathrm{~s}^{-1} \mathrm{Mpc}^{-1}$ (Note: unpublished results from 2015 indicate a slightly different value of $67.1 \mathrm{~km} \mathrm{~s}^{-1} \mathrm{Mpc}^{-1}[7]$. The change does not significantly affect the calculations.)

$$
\begin{aligned}
k & =0 \\
\Omega_{\Lambda} & =\frac{\Lambda}{3 H_{0}}=0.685 \\
\Omega_{r} & =\frac{8 \pi \rho_{r}}{3 H_{0}}=8.35 * 10^{-5} \\
\Omega_{m} & =\frac{8 \pi \rho_{m}}{3 H_{0}}=0.315
\end{aligned}
$$

With the Planck cosmological parameters substituted, equation [3] can be integrated numerically to yield $\eta=3.18$

The co-moving distance $\chi=c \eta$ traversed during the conformal time is similarly 3.18 .

The radius of the observable universe can be calculated to be:

$$
R=\chi R_{H 0}
$$

which yields $14,200 \mathrm{Mpc}=4.63 * 10^{10}$ light-years.

This means that, at the current time, we can effectively observe out to a distance of $4.63 * 10^{10}$ light-years in any directions. Objects beyond this radius are currently unobservable because their signals have not had enough time to reach us. 


\section{The Photon Horizon Radius}

The above value is somewhat optimistic, as it does not take into account that until the recombination era, the universe was opaque to photons. Therefore, to render the limits of the photon horizon (how far light-collecting instruments might observe) we need to adjust the starting time when photons would have been free to traverse space.

Using the relation:

$$
z=\frac{a_{0}}{a}-1
$$

and the value $z_{r e c}=1090$ (provided by Planck), the scale factor at the age of recombination is found to be $1 / 1091 \AA 0.0009$.

Using this as the new upper bound for [3] yields:

$$
\eta_{\text {rec }}=0.0636
$$

Finding the distance to the observable universe from the era of recombination is straightforward:

$$
\chi R_{H 0}=\left(\eta_{0}-\eta_{\text {rec }}\right) R_{H 0}
$$

which equals $13,900 \mathrm{Mpc}$ or $4.53 \times 10^{10}$ light-years.

As mentioned previously, the distance which this calculation provides is based on the use of photons for observation. As current telescopes collect various forms of electromagnetic radiation, photons provide us with the farthest views we can obtain. However, light is, theoretically, not the only method of observation we could use. We are entering a multi-messenger era in astronomy. In the future, researchers might assemble images from neutrinos and other types of particles more transparent to ordinary matter. Hypothetically, we might be able to record and analyze such signals arising from before the era of recombination. The ability to measure these would allow us to increase the radius of our observable universe even further, as we would be able to record the existence of objects even more distant than we could using photons. As our astrophysical knowledge and technologies develop, such possibilities are eagerly anticipated.

\section{Conclusion}

We have updated the 2005 estimates of Gott et al. for the size of the observable universe in accordance with the revised cosmological parameters published by the Planck team in 2013. The value we obtained of $14,200 \mathrm{Mpc}$ for the radius of the observable universe is $0.7 \%$ less than the prior estimate of $14,300 \mathrm{Mpc}$. Similarly, the value we obtained for the radius of the horizon determined by photons emitted during the recombination era is $13,900 \mathrm{Mpc}$, also $0.7 \%$ less than the prior estimate of $14,000 \mathrm{Mpc}$. Therefore, the observable universe is slightly smaller than previously calculated.

\section{References}

1. A. G. Riess, et. al., "Observational Evidence from Supernovae for an Accelerating Universe and a Cosmological Constant," Astronomical Journal, vol. 116, pp. 1009-1038, 1998.

2. S. Perlmutter, et. al., "Measurements of Omega and Lambda from 42 High-Redshift Supernovae," Astrophysical Journal, vol. 517, pp. 565-586, 1999.

3. T. M. Davis and C. H. Lineweaver, "Expanding Confusion: Common Misconceptions of Cosmological Horizons and the Superluminal Expansion of the Universe," Publications of the Astronomical Society of Australia, vol. 21, pp. 97-109, 2004.

4. J. R. Gott III, et. al., "A Map of the Universe," Astronomical Journal, vol. 624, pp. 463-484, 2005.

5. D. N. Spergel, et. al., "First year Wilkinson Microwave Anisotropy Probe (WMAP) observations: determination of cosmological parameters," Astronomical Journal Supplement, vol. 148, pp. 175-194, 2003.

6. P. A. R. Ade, et. al., "Planck 2013 results. XVI. Cosmological Parameters ," Astronomy and Astrophysics, vol. 571, no. A16, pp. 1-66, 2014.

7. P. A. R. Ade, et. al., "Planck 2015 results. XIII. Cosmological parameters," ArXiv:1502.01589. Accepted for publication in Astronomical Journal, 2016. 